\title{
Exploring the impact of a brief mindfulness induction on motor inhibitory control
}

\author{
Satish Jaiswal ${ }^{1,4}$ (D), Shao-Yang Tsai ${ }^{1}$, Chi-Hung Juan ${ }^{1}$, Wei-Kuang Liang ${ }^{1}$ and Neil G. Muggleton ${ }^{1,2,3, *}$ \\ ${ }^{1}$ Institute of Cognitive Neuroscience, National Central University, No.300, Jhongda Road., Jhongli county, Taoyuan City - \\ 32001, Taiwan, ${ }^{2}$ Institute of Cognitive Neuroscience, University College London, London, UK, ${ }^{3}$ Department of Psychology, \\ Goldsmiths, University of London, London, UK, and ${ }^{4}$ Department of Pathology and Laboratory Medicine, School of Medicine \\ and Public Health, University of Wisconsin-Madison, Madison, WI. \\ ${ }^{*}$ Corresponding author: Email: neil.muggleton@gmail.com
}

(Received 11 October 2019; Revised 28 May 2020; Accepted 29 May 2020)

\begin{abstract}
Inhibitory control can be divided into motor and cognitive inhibition. The current research is the first study exploring the impact of brief mindfulness training on motor inhibition, measured by a stop signal task in participants without any meditation experience. Motor inhibition performance was compared before and immediately after three different conditions; a brief mindfulness induction, a resting state and an active control session in which participants listened to their favorite music. Post-test learning effect on go-reaction times was seen for the resting and mindfulness conditions, but was absent in the music session, possibly due to emotional arousal might have led slower responses. Brief mindfulness training did not significantly alter inhibitory control, although marginal improvement in stop signal reaction time following the mindfulness induction was observed. Motor inhibition appears unresponsive to either short-term or long-term mindfulness practice. Future mindfulness studies should explore a broad spectrum of cognitive functions and populations.
\end{abstract}

Keywords: mindfulness; preferred music; resting state; motor inhibition; stop signal task

\section{Introduction}

Inhibitory control of an ongoing action is critical for survival. Additionally, Moffitt et al. (2011) reported children with low self-control scores experience various socioeconomic problems later in life. While increased mindfulness may potentially result in better self-control, to date, few studies have explored its effects on motor inhibition. A potentially useful task for this is the go-stop paradigm (Heeren et al., 2009). However, this has limitations, including confounds with short-term memory and a potentially high probability of having to withhold a response. An alternative, the stop signal task (Logan, 1994), has only imperative signals, such as arrows, with stop trials differing from go signals in that the imperative signal is followed by a visible indicator as the stop signal. Performance on this task is explained by a 'horse-race' model that assumes that there is a competition between pre-potent go and stopping responses to reach the threshold and determining the response.

\footnotetext{
(c) The Author(s), 2020. Published by Cambridge University Press. This is an Open Access article, distributed under the terms of the Creative Commons Attribution licence (http://creativecommons.org/licenses/by/4.0/), which permits unrestricted re-use, distribution, and reproduction in any medium, provided the original work is properly cited.
} 


\section{Objective}

A within subject, pre-test versus post-test design was employed, with effects of brief mindfulness induction compared to effects of a well-controlled resting state and an active control in which participants listened to their favorite music. It was predicted that the participants' speed on an imperative go-signal (Go-RT) would be faster for post-test versus pre-test due to a practice effect. The primary measure of inhibitory control, stop signal reaction time (SSRT) was predicted to be shorter in the posttest condition following brief mindfulness induction in comparison to both the resting and preferred music conditions, indicating better motor inhibition facilitated by mindfulness.

\section{Methods}

Thirty participants (13 females) were recruited from the National Central University with a mean age of $23.1(\mathrm{SD}=2.2)$ years. None had any prior meditation experience nor any neuropsychiatric disorder or current medication. All participants gave written informed consent in accordance with the Declaration of Helsinki and all experimental procedures were approved by the Institutional Review Board of National Taiwan University, Taipei, Taiwan. The stop signal task (see Figure 1) was performed by each participant in a pre-test and a post-test for three different conditions, each on different days (Figure 2). These conditions were resting state, listening to preferred music and engagement in mindfulness induction, all 20-minute duration. Resting state involved sitting idle, the music condition involved listening to their favorite music on their cellphones and for the mindfulness condition they followed instructions presented via a Power Point file (for details see Supplementary Document (dx.doi.org/10.17504/protocols.io.74ihque)).

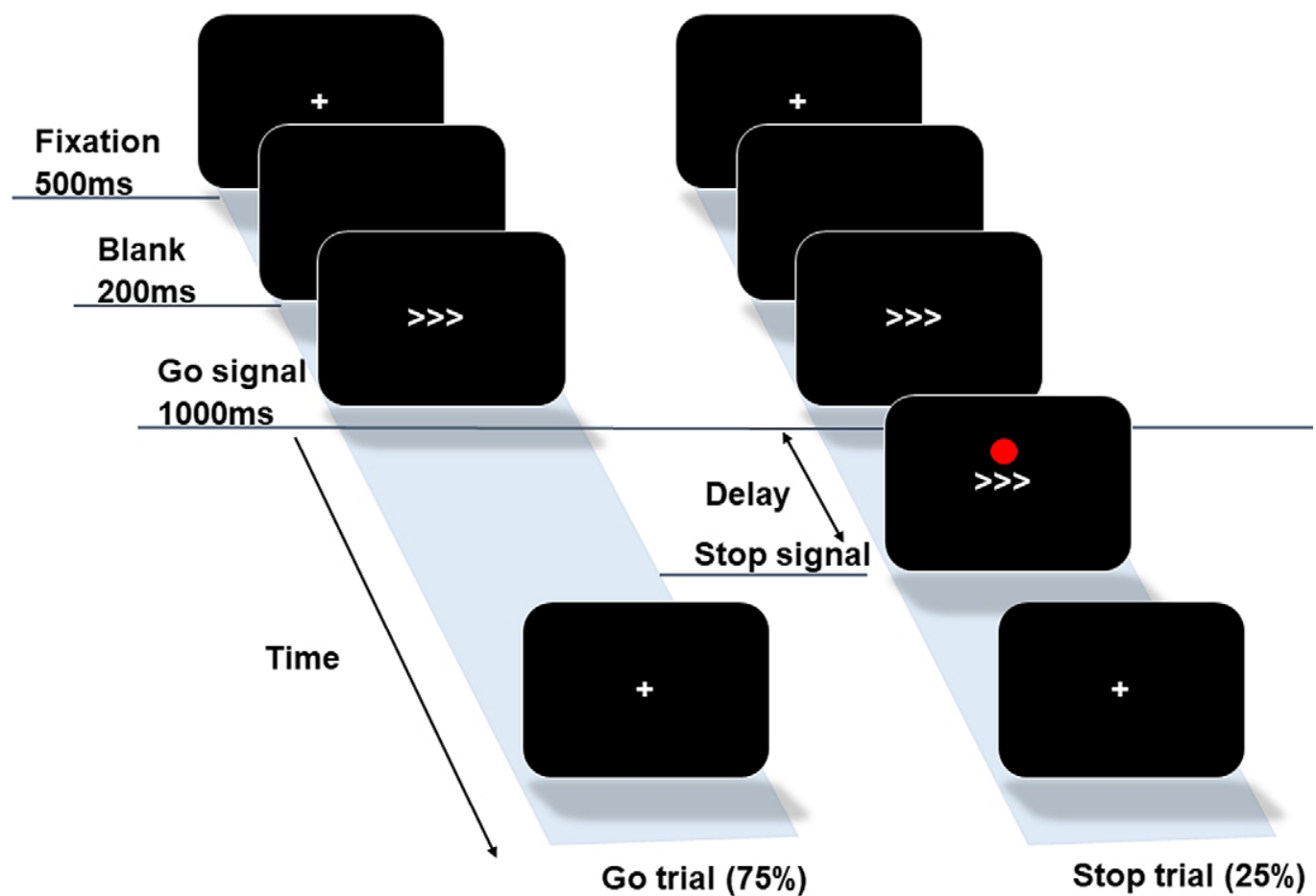

Figure 1. Stop Signal Task. In go trials, a central fixation cross '+' was displayed for 500 milliseconds (ms) and was followed by a blank screen for $200 \mathrm{~ms}$. Next, a go stimulus was presented for $1,000 \mathrm{~ms}$, to which a response is typically required, with participants responding to left arrows by pressing the ' $X$ ' key and to right arrows by pressing the ' $M$ ' key on a computer keyboard with either their left or right index fingers, respectively. Each trial was followed by a blank screen inter-trial interval for a duration between $500 \mathrm{~ms}$ and $1,000 \mathrm{~ms}$. In stop trials, a red dot was presented as a stop signal positioned above the go stimulus location, with the presentation of these trials otherwise identical to the go trials. On trials where the stop signal was presented, participants were required to withhold their responses to the go signal. 


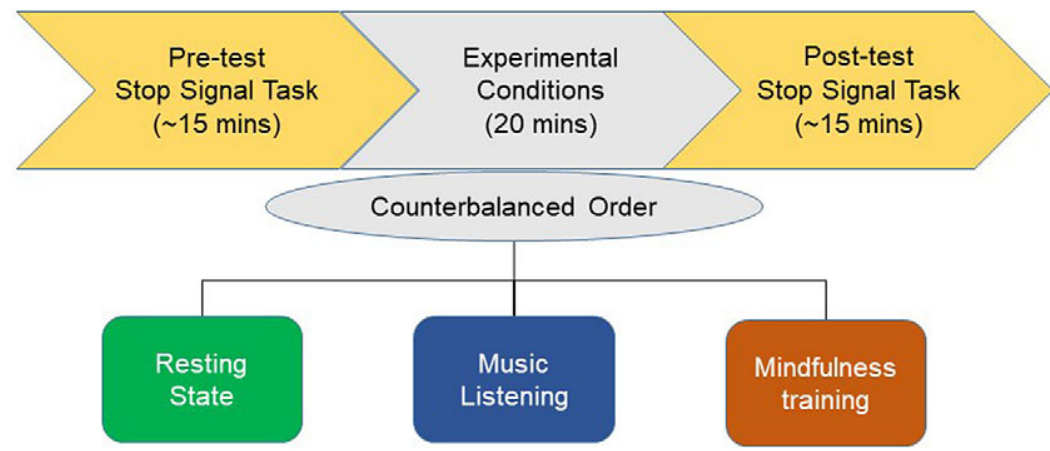

Figure 2. Study Design In this within-subject design, the stop signal task was performed by each participant before and after each of three different conditions. These conditions were resting state, listening to preferred music and engagement in mindfulness induction. Each condition was 'presented' for 20 minutes following the pre-test on three different days. The order of the conditions was counterbalanced across participants.

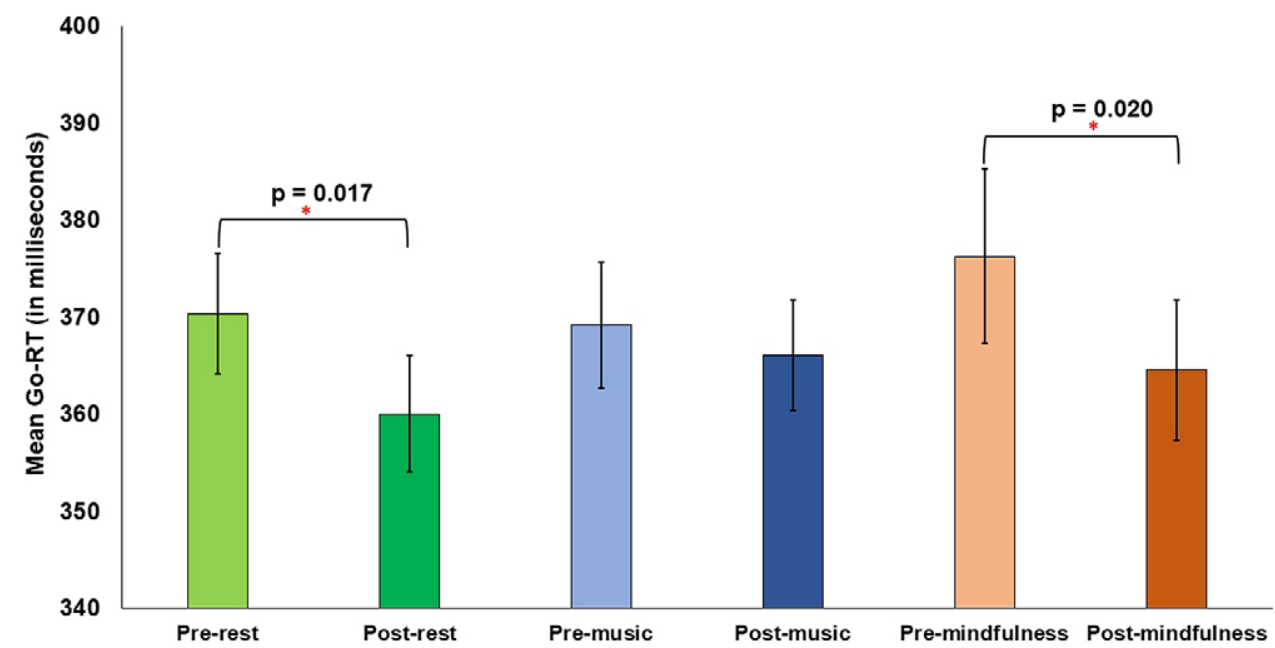

Figure 3. Go-RTs across conditions and sessions. Significant improvement, likely due to a practice effect, was seen post rest and mindfulness inductions, but not following listening to preferred music (error bars=standard errors of the mean).

\section{Results}

Two participants were excluded, having RTs greater than \pm 3 SD from the mean. Two-way repeated measures ANOVA was used for Go-RTs and SSRTs. Go-RTs showed no significant effect of condition $[\mathrm{F}(2,54)=0.121, \mathrm{p}=0.886]$ or condition $\mathrm{x}$ session interaction $[\mathrm{F}(2,54)=1.275, \mathrm{p}=0.288]$, but a significant effect of session $[\mathrm{F}(1,27)=13.150, \mathrm{p}=0.001]$. Ad hoc pair-wise t-tests showed improvement across sessions for resting and mindfulness conditions, but not for the music condition $[\mathrm{t}(27)=1.176, \mathrm{p}=$ 0.250] (Figure 3). SSRTs showed no significant effect of condition $[F(2,54)=0.371, p=0.692]$, session $[\mathrm{F}(1,27)=1.385, \mathrm{p}=0.250]$, or interaction between condition and $\operatorname{session}[\mathrm{F}(2,54)=0.946, \mathrm{p}=0.395]$. Ad hoc paired-tests showed the mindfulness condition resulted in lower SSRTs that approached significance for post-test versus pre-test $[\mathrm{t}(27)=1.868, \mathrm{p}=0.073]$ (Figure 4).

\section{Discussion}

Post-test Go-RTs in mindfulness and resting condition showed an expected practice effect (Ando et al., 2002). However, no such speed enhancement was seen for the preferred music condition. It is possible 


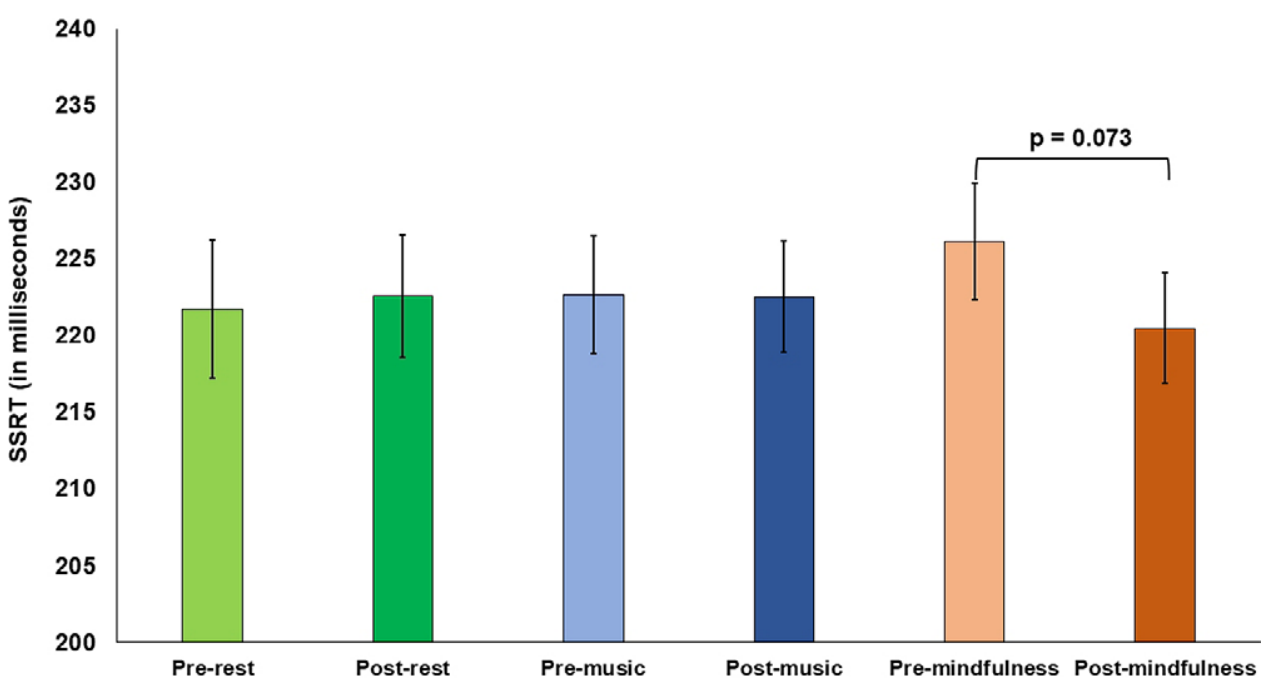

Figure 4. SSRTs across conditions and sessions. The mindfulness induction was associated with a marginal reduction in SSRT, but resting and active control (music) conditions did not show any significant change in post-test sessions (error bars = standard error of the mean).

that the music listening may have caused emotional and physiological arousal (Rickard, 2004). DroitVolet et al. (2013) found altered time perception due to emotional effects of music, mainly regulated by the temporal dynamics of the music. A marginal trend for slower SSRTs in the post-test session for the mindfulness condition might be linked with facilitated inhibition could be due to a lack of statistical power.

\section{Conclusion}

Consistent with a prior longitudinal study comparing mindfulness meditators and matched controls (Heeren et al., 2009), no difference in motor inhibition was observed here for the mindfulness condition compared to the two control conditions. The data obtained here are consistent with motor inhibitory control being unaffected by mindfulness, irrespective of whether engagement with the technique is long or brief. Future mindfulness studies should investigate a broader spectrum of cognitive tasks and sample populations (Jaiswal et al., 2018) to test cognitive functions and population types that might be sensitive to mindfulness interventions.

Author Contributions. SJ and NGM conceived and designed the study. SJ conducted data gathering. SYT and WKL assisted in statistical analyses. SJ, NGM and CHJ wrote the article.

Funding. This work was sponsored by the Ministry of Science and Technology, Taiwan (grant numbers: 107-2420-H-008-009-; 108-2639-H-008-001-ASP; 108-2321-B-075 -004 -MY2;107-2628-H-008 -002 -MY3; 106-2410-H-008-038-MY3; 106-2628H-008-002-MY4; 107-2410-H-008 -040 -MY3) and sponsored by Taiwan Ministry of Education's “Academic Strategic Alliance: Taiwan and Oxford University" project grant (MOE Oxford- NCU-BRC collaborative project).

Conflicts of Interest. The authors have none to declare.

Data Availability. The dataset can be accessed at Zendo using the doi provide below: https://doi.org/10.5281/zenodo.3483190 Supplementary Materials. To view supplementary material for this article, please visit http://dx.doi.org/10.1017/exp.2020.29.

\section{References}

Ando, S., Kida, N., \& Oda, S. (2002). Practice effects on reaction time for peripheral and central visual fields. Perceptual and Motor Skills, 95, 747-751. doi:https://doi.org/10.2466\%2Fpms.2002.95.3.747. 
Droit-Volet, S., Bueno, L. J., \& Bigand, E. (2013). Music, emotion, and time perception: The influence of subjective emotional valence and arousal?. Frontiers in Psychology, 17, 417. https://doi.org/10.3389/fpsyg.2013.00417.

Heeren, A., Van Broeck, N., \& Philippot, P. (2009). The effects of mindfulness on executive processes and autobiographical memory specificity. Behaviour Research and Therapy, 47, 403-409. https://doi.org/10.1016/j.brat.2009.01.017.

Jaiswal, S., Tsai, S. Y., Juan, C. H., Liang, W. K., \& Muggleton, N. G. (2018). Better cognitive performance is associated with the combination of high trait mindfulness and low trait anxiety. Frontiers in Psychology, 9, 627. https://doi.org/10.3389/ fpsyg.2018.00627.

Logan, G. D. (1994). On the ability to inhibit thought and action: A users' guide to the stop signal paradigm. https:// psycnet.apa.org/record/1994-97487-005

Moffitt, T. E., Arseneault, L., Belsky, D., Dickson, N., Hancox, R. J., Harrington, H., Houts, R., Poulton, R., Roberts, B. W., Ross, S., \& Sears, M. R. (2011). A gradient of childhood self-control predicts health, wealth, and public safety. Proceedings of the National Academy of Sciences, 108, 2693-2698. https://doi.org/10.1073/pnas.1010076108.

Rickard, N. S. (2004). Intense emotional responses to music: A test of the physiological arousal hypothesis. Psychology of Music, 32, 371-388. https://psycnet.apa.org/doi/10.1177/0305735604046096.

Cite this article: Jaiswal S, Tsai S-Y, Juan C-H, Liang W-K, Muggleton NG (2020). Exploring the impact of a brief mindfulness induction on motor inhibitory control Experimental Results, 1, e24, 1-8. https://doi.org/10.1017/exp.2020.29 


\title{
Peer Reviews
}

\author{
Reviewing editor: Dr. Elissa Aminoff
}

Fordham University, Psychology, Dealy Hall 332, 441 E. Fordham Rd, New York, New York, United States, 10458

doi:10.1017/exp.2020.29.pr1

\section{Review 1: Exploring the impact of a brief mindfulness induction on motor inhibitory control}

Reviewer: Dr. Melissa Birkett

Southern Oregon University, Psychology, 1250 Siskiyou Blvd, Ashland, Oregon, United States, 97520-5010

Date of review: 02 February 2020

Conflict of interest statement. Reviewer declares none.

Comments to the Author: The authors present the results of an experiment comparing pre and post response times to go- and no-go-type tasks before/after three, 20-min conditions: resting control, mindful meditation and active control/listening to music. Post-condition reaction time was significantly reduced in the control and meditation conditions for the go task only. The experiment provides novel experimental support for prior meditation/reaction time studies. Strengths of the study include using meditation-naive participants, counterbalancing conditions, and including a concise and clear manuscript and supplemental files. Specifying the number of pre and post-task trials for each task, relevant effect sizes, and reporting any subjective feedback from participants about the conditions (for example, rating the experience of meditation, rest or music) may be helpful for additional interpretation. One challenge to interpreting the null results in the active control condition is the unstandardized format of the music condition. For example, music was self-selected by participants and played on cell phones. Specifying control for volume, speakers/earphones, number of songs and emotional content could additionally help in interpreting the results. An additional brief explanation of the role of the music condition in the study (as a form of active control) might also be helpful. Overall, the results of this paper help strengthen the understanding of mindfulness meditation on motor inhibitory control.

\section{Score Card}

Presentation

Is the data presented in the most useful manner? (40\%)

Does the paper cite relevant and related articles appropriately? (30\%)

\section{Context}

Does the title suitably represent the article? (25\%)

Does the abstract correctly embody the content of the article? (25\%)

Does the introduction give appropriate context? (25\%)

Is the objective of the experiment clearly defined? (25\%) 
Are the limitations of the experiment as well as the contributions of the experiment clearly outlined? (20\%) 


\section{Review 2: Exploring the impact of a brief mindfulness induction on motor inhibitory control}

Reviewer: Prof. Yvonne N. Delevoye-Turrell ${ }^{1,2}$ (iD

${ }^{1}$ University of Lille, Psychology, rue du Barreau, Lille, France, 59000

${ }^{2}$ CNRS, UMR 9193, Paris, France, 75794

Date of review: 08 May 2020

Conflict of interest statement. Reviewer declares none

Comments to the Author: The present paper describes the exploration of the impact of brief mindfulness training on motor inhibition. The study is well described but there is no power calculation for the statistics. The choice of the paradigms is well explained but there are too few. The biggest drawback stands within the absence of significant results. In addition:

1- the pre-test baseline level for the SSRT in the mindfulness group is greater than that observed in the two control conditions;

2- the SSRT in the mindfulness group is not significantly lower in the post-compared to the pre-session.

Hence, as it stands, there is an absence of results.

In closing, there is a problem in the selection of the participants. Indeed, mindfulness practice is not easy. For it to have any impact on health or on general well-being, regular practice is required. Hence, for science to be able to demonstrate quantitative impact of such a qualitative practice, I think that the authors should have conducted the study with adults characterized with at least an intermediate knowledge of mindfulness practice.

In conclusion, even if I find the topic very interestig, I have no choice than to reject the study for publication.

\section{Score Card}

Presentation

3.1

Is the article written in clear and proper English? (30\%)

Is the data presented in the most useful manner? (40\%)

Does the paper cite relevant and related articles appropriately? (30\%)

Context

Does the abstract correctly embody the content of the article? (25\%)

Does the introduction give appropriate context? (25\%)

Is the objective of the experiment clearly defined? (25\%)

Analysis

Are the limitations of the experiment as well as the contributions of the 